\title{
Probabilistic Analysis of Long-Term Swarm Performance under Spatial Interferences
}

\author{
Yara Khaluf $^{1}$, Mauro Birattari ${ }^{2}$, and Franz Rammig ${ }^{1}$ \\ 1 Heinz Nixdorf Institut, University of Paderborn \\ Fürstenallee 11, 33102 Paderborn, Germany \\ \{yara,franz\}@hni.uni-paderborn.de \\ https://www.hni.uni-paderborn.de/ \\ 2 IRIDIA, Université Libre de Bruxelles \\ B-1050 Brussels, Belgium \\ mbiro@ulb.ac.be \\ http://iridia.ulb.ac.be
}

\begin{abstract}
Swarm robotics is a branch of collective robotics that outperforms many other systems due to its large number of robots. It allows for performing several tasks that are beyond the capability of a single or multi robot systems. Its global behaviour emerges from the local rules implemented on the level of its individual robots. Thus, estimating the obtained performance in a self-organized manner represents one of the main challenges, especially under complex dynamics like spatial interferences. In this paper, we exploit the central limit theorem (CLT) to analyse and estimate the swarm performance over long-term deadlines and under potential spatial interferences. The developed model is tested on the well-known foraging task, however, it can be generalized to be applied on any constrictive robotic task.
\end{abstract}

Keywords: Swarm robotics, Time-constrained tasks, Central limit theorem.

\section{Introduction}

Swarm robotics is a high density multi-robot system, where the global behaviour emerges from local rules implemented on the level of individual robots. These systems are characterized by a set of advantages including: redundancy, scalability and flexibility which introduce them as a promising approach for a large spectrum of tasks.

Spatial interferences, on the other hand, affect significantly the performance of the single robot and consequently the collective performance of the swarm. A well-studied example is the foraging, where robots are exploited to retrieve scattered objects to a special area called "nest". As noted in [5]10, the increment of the robots' number in a task like foraging, decreases the performance of a single robot which represents the number of retrieved objects per time unit. In the case of swarm performance, it may increase by adding robots up to an 
optimal number and after that it starts to decrease affected by the interferences among robots. Although the influence of spatial interferences among robots has been studied on a limited number of swarm scenarios, mainly on foraging, the observations do not appear to be surprising. Increasing the number of robots, increases the time required by the single robot to accomplish individual parts of the task because of the concurrence among robots. This decreases in turn, the number of parts that could be accomplished by single robots within a specific time period. From the swarm point of view, the obtained performance keeps increasing as long as the benefit of parallelising the work is still larger than the time penalty paid because of the interferences. Swarm robotic systems are prone to intensive spatial interferences and as robotic missions are generally characterized by their long-term durations, providing the ability to estimate the swarm performance for long-term tasks and under the spatial interferences is of a significant importance. However, performing this estimation by running real experiments or computer simulations is intensive time and resource consuming. Consequently, alternative tools are required to perform such kind of estimations. In this paper, we investigate the use of the Central Limit Theorem (CLT) as a tool for approximating the swarm performance and analysing it probabilistically over long-term deadlines and under robots spatial interferences. Central limit theorem, in its classic version, states that the mean of a sufficiently large set of independent and identically distributed random variables each with a finite mean and variance tends to be distributed normally. How large the set of i.i.d. should be, is based on their distribution parameters.

The rest of the paper is organized as following: Section 2 lists a set of related work. Section 3 formulates the problem of the swarm performance estimation over long-term deadlines. In Section 4 The central limited theorem is introduced and the proposed estimator model for the swarm performance is illustrated. Section 5 presented a foraging scenario to verify the proposed estimation strategy and Section [6] concludes the paper.

\section{Related Work}

Swarm robotic performance is influenced by the interferences among participating robots [2. Most of the performed studies were focusing on characterizing how the amount of work accomplished within a specific time unit, changes by changing the number of working robots. The studies were mostly accomplished on well-known swarm tasks like foraging and the conclusions were similar, namely, that increasing the number of robots decreases the performance of individual robots. After characterizing the relation between the swarm size and performance, several studies were performed to improve the swarm performance by reducing the density of spatial interferences. In [1] several types of interferences in multi-robot systems have been defined and it presented the interactions among robots working together in a common area, like the nest, as the main type of robots interactions. The authors have proposed two techniques to arbitrate the impact of interactions. First, by making sure that robots are working in different 
areas and second, by scheduling the occupation of shared areas. The first proposal was further investigated under the term bucket-brigade like in [15, 16] and [10], in addition to [4], where the approach was extended to consider adaptive working areas. Task partitioning represents another technique, which is used to improve the swarm performance under spatial interferences. In $[12$ a task partitioning technique was proposed, in which the shared area was divided into two areas and the robots select their area using a threshold mechanism. In [13, the authors studied the role of task partitioning in reducing the concurrent access to the nest area in a harvesting task.

On the other hand, characterizing swarm performance by means of real experiments is not always possible and is an expensive solution from both time and hardware points of view. In addition, computer simulations are very time consuming especially when tasks are associated with long-term deadlines. In such cases, the mathematical modelling represents one of the best approaches. A mathematical model has been introduced in [5], which characterizes the performance of the single robot and the swarm under spatial interferences. In [9] a list of the various mathematical models which can be applied in swarm systems, is reported. Most of these mathematical studies were focusing on specific swarm scenarios like foraging in [7] or collaborative distributed manipulation in [8]. To our best knowledge no study was focusing on the mathematical analysis of the collective swarm performance within specific deadlines. In addition, the probability analysis of swarm robotics was not considered intensively and only few studies were performed in that field like in 6]. The central limit theorem [14, is a wide-applied theorem in many fields related to measurement approximations and hypothesis testing. However, it is not investigated yet within the context of swarm robotics. This important theorem is exploited, here, to develop swarm performance estimators which allow for a probabilistic characterization of swarm performance over long-term deadlines and under the dynamics of spatial interferences.

\section{Problem Formulation}

We consider constructive tasks where the total contribution on the task within a particular time period is the sum of the individual contributions of robots over that time period. Each of the considered tasks can be characterized by its long-term deadline, that represents the time point after which the robots should stop to work on the task.

In swarm robotic systems the contribution of a single robot on any task is a random variable which can belong to the discrete, as well as, to the continuous space based on the type of the task. In a task like pushing a box, the robot performance is a continuous random variable represents the distance the box travels within a specific time unit. However, in a foraging task the robot performance represents the number of retrieved objects and belongs to the discrete space. In this paper, we focus on the discrete space of robot performance where the task consists of discrete parts to be accomplished.

Let us assume a task $T_{i}$ with the deadline $D_{i}$ and a homogeneous swarm of $N$ simple robots. Each of these robots is able to accomplish one part of $T_{i}$ 
at a time. We use $\beta_{i j}\left(D_{i}\right)$ to denote the discrete random variable associated with the number of parts can be accomplished by the robot $R_{j}$ on task $T_{i}$ up to its deadline of $D_{i}$, under the influence of spatial interferences. The swarm performance on task $T_{i}$ up to the deadline $D_{i}$ is denoted by $\omega_{i}\left(D_{i}\right)$. Thus, $\omega_{i}\left(D_{i}\right)$ is the total number of parts accomplished by the swarm within the time of $D_{i}$ under the spatial interferences and is calculated as the sum of the robots' individual contributions:

$$
\begin{aligned}
\omega_{i}\left(D_{i}\right) & =\beta_{i 1}\left(D_{i}\right)+\beta_{i 2}\left(D_{i}\right)+\ldots+\beta_{i N}\left(D_{i}\right) \\
& =\sum_{j=1}^{N} \beta_{i j}\left(D_{i}\right)
\end{aligned}
$$

We divide the time period between the start of the execution $t=0$ and the task deadline $D_{i}$ into equal and non-overlapping time-windows each with the length $\tau$. The length $\tau$ of the time-window is selected under the following constraints: It should be equal to or greater than the average time required by a single robot to accomplish one part on task $T_{i}$, The task deadline $D_{i}$ should be a multiplier of $\tau$ and $\tau$ should be significantly smaller than the task deadline: $\tau \ll D_{i}$.

The swarm performance at deadline $D_{i}$ is the sum of the swarm contributions over all the time-windows included within the deadline $D_{i}$. Hence, we can calculate the swarm performance at the deadline $D_{i}$ as in following:

$$
\begin{aligned}
\omega_{i}\left(D_{i}\right) & =\omega_{i}\left(\tau_{1}\right)+\omega_{i}\left(\tau_{2}\right)+\ldots+\omega_{i}\left(\tau_{K}\right) \\
& =\sum_{j=1}^{K} \omega_{i}\left(\tau_{j}\right)
\end{aligned}
$$

where $K$ is the number of time-windows included in deadline $D_{i}$.

On the other hand, the swarm performance at deadline $D_{i}$ is the sum of the individual robots' contributions over all the time-windows included in the deadline $D_{i}$. Using Equation (11) the swarm performance can be calculated in terms of the individual robots' contributions as in following:

$$
\begin{aligned}
\omega_{i}\left(D_{i}\right) & =\left(\beta_{i 1}\left(\tau_{1}\right)+\beta_{i 2}\left(\tau_{1}\right)+\ldots+\beta_{i N}\left(\tau_{1}\right)\right) \\
& +\ldots \\
& +\left(\beta_{i 1}\left(\tau_{K}\right)+\beta_{i 2}\left(\tau_{K}\right)+\ldots+\beta_{i N}\left(\tau_{K}\right)\right) \\
& =\sum_{j=1}^{K} \sum_{l=1}^{N} \beta_{i l}\left(\tau_{j}\right)
\end{aligned}
$$

The goal is to estimate the performance which can be obtained by a swarm of $N$ robot at the deadline $D_{i}$ and under the influence of spatial interferences. We aim to perform this estimation with the minimum time and resources consumption by launching short-time real experiments or computer simulations. The estimation is carried out in a probabilistic manner, where we derive the probability density function (PDF) in addition to the cumulative distribution function (CDF) of 
the random variable that is associated with the swarm performance. Such a probabilistic analysis helps us to answer questions like: "What is the probability of achieving a specific swarm performance $S_{i}$ within the deadline $D_{i}$ under the influence of spatial interferences?".

$$
\operatorname{Pr}\left(\omega_{i}\left(D_{i}\right) \geqslant S_{i}\right)
$$

\section{Probabilistic Analysis of Swarm Performance under Spatial Interferences}

In this section, we investigate the well-known central limit theorem in performing a probabilistic analysis of the swarm performance at long-term deadlines under the influence of spatial interferences. Central limit theorem is a wide-applied theorem specially in fields of hypothesis testing, cancelling of communication noise and statistic 3 . Let us have a set of $n$ independent and identically distributed random variables $\left\{X_{1}, X_{2}, \ldots, X_{n}\right\}$, sampled from a distribution with a specific mean $\mu$ and a variance $\sigma^{2}$. The central limit theorem (CLT) in its classic version states that for sufficiently large $n$, the sum of the $n$ random variables is normally distributed and can be characterized with the following mean and variance:

$$
\mu_{n}=n \mu \quad(5) \quad \sigma_{n}^{2}=n \sigma^{2}
$$

The swarm contribution $\omega_{i}\left(\tau_{j}\right)$ over the time-window $\tau_{j}$, is the random number of parts accomplished by the swarm on task $T_{i}$ within the time-window $\tau_{j}$. Concurrently, the single robot contribution $\beta_{i l}\left(\tau_{j}\right)$ is the random number of parts accomplished by a single robot on task $T_{i}$ within the time-window $\tau_{j}$. The mean and the variance associated with these two random variables are influenced directly by the number of robots working on the task in addition to the work density available on this task. The work density here refers to the number of parts available on the task and need to be accomplished by the robots. Swarm systems are generally characterized by their large sizes, thus the failure of a few robots or the addition of another few, will not apply a considerable change on the average performance of the swarm or of the single robot. In addition applying the central limit theorem is constrained by having a sufficiently large $n$, where $n$ represents the number of robots in case the swarm performance is defined based on Equation (3). Hence, a small change in the number of robots will not change significantly the total performance of the swarm. On the other hand, the assumption of having a constant work density is associated with a large set of real-world applications. Examples to these tasks could be recycling systems where the robots are responsible to retrieve objects excreted continuously at specific locations to some recycling destination. Another example could be a production-transport system, where objects are assumed to be produced continuously at different locations and require to be transported to specific delivery points.

Based on the above discussion we can describe the two conditions of having a constant average of robots number and a constant work density as realistic assumptions. Let us denote the mean and the standard deviation of the random variable associated with the swarm performance $\omega_{i}(\tau)$ on task $T_{i}$ within the 
time-window $\tau$ respectively by: $\mu_{\omega_{i}(\tau)}$ and $\sigma_{\omega_{i}(\tau)}$. Based on the central limit theorem the long-term swarm performance will be normally distributed:

$$
\omega_{i}\left(D_{i}\right) \sim \operatorname{Norm}\left(K \mu_{\omega_{i}(\tau)}, K \sigma_{\omega_{i}(\tau)}^{2}\right)
$$

On the other hand, we denote the mean and the standard deviation of the random variable associated with the single robot performance $\beta_{i j}(\tau)$ respectively by: $\mu_{\beta_{i j}(\tau)}$ and $\sigma_{\beta_{i j}(\tau)}$. According to the central limit theorem, the long-term swarm performance will be also normally distributed:

$$
\omega_{i}\left(D_{i}\right) \sim N o r m\left(K N \mu_{\beta_{i j}(\tau)}, K N \sigma_{\beta_{i j}(\tau)}^{2}\right)
$$

Consequently, the swarm performance can be characterized probabilistically by using the cumulative distributed function (CDF) of the normal distribution, which for the mean $\mu$ and the variance $\sigma^{2}$, is defined as in following:

$$
\operatorname{Pr}(X \leqslant x)=\frac{1}{2}+\frac{1}{2} \operatorname{erf}\left(\frac{x-\mu}{2 \sigma^{2}}\right)
$$

We substitute the random variable $X$ by the swarm performance $\omega_{i}\left(D_{i}\right)$ and the value of small $x$ by a desired performance $S_{i}$, that represents the number of parts to accomplish up to the task deadline $D_{i}$. The probability we are interested to calculate is the one in Equation (4): $\operatorname{Pr}\left(\omega_{i}\left(D_{i}\right) \geqslant S_{i}\right)$, which can be expressed using the CDF of the normal distribution (9):

$$
\begin{aligned}
\operatorname{Pr}\left(\omega_{i}\left(D_{i}\right) \geqslant S_{i}\right) & =\operatorname{Pr}\left(\omega_{i}\left(D_{i}\right)>\left(S_{i}-1\right)\right) \\
& =1-\operatorname{Pr}\left(\omega_{i}\left(D_{i}\right) \leqslant\left(S_{i}-1\right)\right)
\end{aligned}
$$

Consequently, the central limit theorem (CLT) can be applied to estimate the long-term performance of swarm robotics, efficiently, in both following contexts:

- Swarm performance over short experiments: the swarm performance at deadline $D_{i}$ is the sum of the swarm contributions over all time-windows included within the deadline $D_{i}$. We map each of these swarm contributions to a random variable with the mean $\mu_{\omega_{i}(\tau)}$ and the variance $\sigma_{\omega_{i}(\tau)}^{2}$ which are measured over one experiment of the length $\tau$. Consequently, the central limit theorem can be applied to approximate the swarm performance at $D_{i}$ as the sum of these random variables, like in Equation (2).

- Single robot performance over short experiments: the swarm performance at deadline $D_{i}$ can be calculated as the sum of the individual robots' contributions over all time-windows included within the deadline $D_{i}$. As the single robot performance over one time-window experiment can be measured by the robot itself, this estimation technique represents a "self-organized" one. The robot works on the task for one time-window to estimate the mean and the standard deviation of the random time required to accomplish one part of that task. After that, the central limit theorem is applied to approximate the swarm performance at $D_{i}$ using Equation (3). 


\section{Scenario and Evaluation}

We consider the foraging scenario, where a large number of objects are scattered uniformly over an object-area and need to be retrieved by a swarm of robots to some nest-area. During the experiment, each robot can be in one of the following states: exploring or retrieving. A robot being in the exploring state searches for objects to retrieve and as soon as an object is found, the robot changes its state to retrieving and starts to move towards the nest-area. It is assumed that new objects are popping up in the arena and the objects density remains constant throughout the whole experiment. The ARGoS1 simulator [11] is used to calculate an average performance function via repeated high-level simulations in order to characterize the effect of the spatial interferences on the performance of the single robot as well as on the swarm. The simulations are repeated for 125 times. Figure 1 shows how the mean of the single robot performance decreases by increasing the number of robots in addition to the standard deviation of this performance for different swarm sizes. Figure 2 shows the change in the mean of the swarm performance and its standard deviation while applying the same increment in the swarm size. We consider the foraging scenario illustrated in Section 5 , where it is carried out by a swarm of 30 robots within the deadline $D_{i}=12 \cdot 10^{3}$ seconds. The time-window length is set to $\tau=100$. Figure 3 shows the mean $\mu$ in addition to the $3 \times \sigma$ of the random number associated with the retrieved objects over all time-windows up to the $D_{i}=12 \cdot 10^{3}$ seconds. Figure 4 shows the time it takes the average of the system performance to stabilize. This time is referred to as start-up time. At the beginning of the foraging task all robots are free to search for objects and to retrieve them as soon as they find any. Thus, the number of objects retrieved at the beginning is higher than the number will be retrieved later on, when the robots are divided between robots which are searching and free to retrieve and robots which are retrieving. This is the reason behind the existence of such a start-up time after which the system performance stabilizes. The accuracy of the CLT estimation of the swarm performance is influenced by including the system performance during the start-up time or excluding it. This influence varies based on the relative relation between the length of both: the deadline $D_{i}$ and the start-up time. The swarm performance will be estimated within the two contexts mentioned above. First by using the swarm contributions and second using the individual robot's contribution both measured over short experiments.

\section{- Swarm performance over short experiments:}

We use the swarm contribution achieved by the whole swarm within one time-window $\tau=100$ second. We substitute it in Equation (2), where the deadline of $D_{i}=12 \cdot 10^{3}$ includes 120 time-window of the length 100 seconds:

$$
\omega_{i}\left(D_{i}\right)=\sum_{j=1}^{K} \omega_{i}\left(\tau_{j}\right) \quad \Rightarrow \quad \omega_{i}\left(12 \cdot 10^{3}\right)=\sum_{j=1}^{120} \omega_{i}(100)
$$

\footnotetext{
${ }^{1}$ ARGoS is a discrete-time physics-based simulation framework developed within the Swarmanoid project. It can simulate various robots at different levels of details, as well as a large set of sensors and actuators.
} 


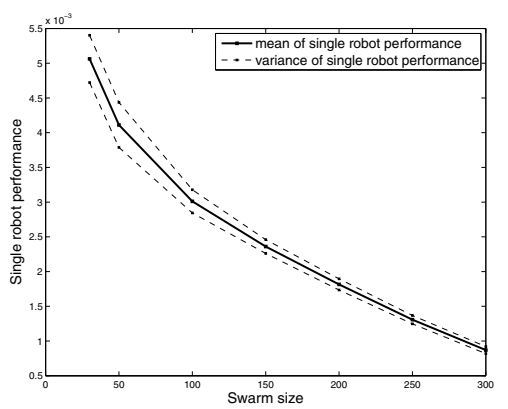

Fig. 1. Single robot performance under spatial interferences during 1 second

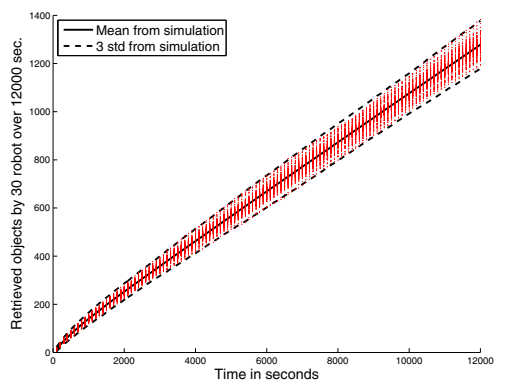

Fig. 3. $\mu$ and $3 \times \sigma$ of the number of objects retrieved during $12 \cdot 10^{3}$ seconds

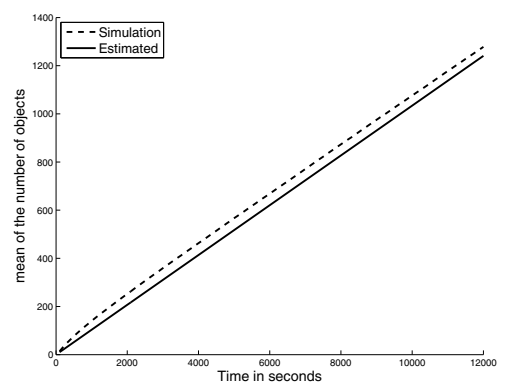

Fig. 5. Mean of retrieved objects throughout $2 \cdot 10^{3}$ seconds vs. the mean predicted by CLT

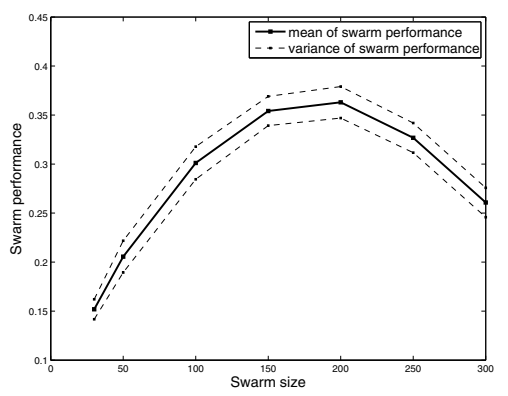

Fig. 2. Swarm performance under spatial interferences during 1 second

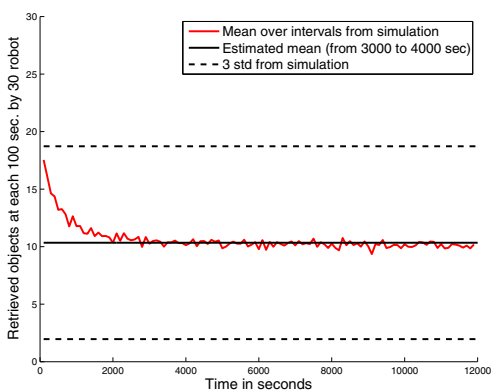

Fig. 4. $\mu$ and $3 \times \sigma$ of the number of objects retrieved over all 100 seconds intervals included in the deadline $D_{i}$

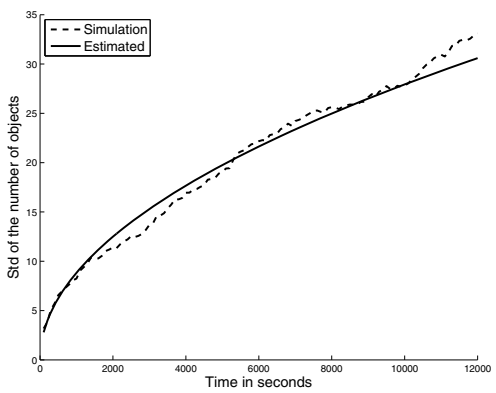

Fig. 6. Standard deviation of retrieved objects throughout $2 \cdot 10^{3}$ seconds vs. the mean predicted by CLT 
According to the central limit theorem, the r.v. associated with the number of retrieved objects at the deadline $12 \cdot 10^{3}$ is normally distributed with the mean and the standard deviation as in following:

$$
\mu_{\omega_{i}\left(12 \cdot 10^{3}\right)}=120 \mu_{\omega_{i}(100)} \quad(12) \quad \sigma_{\omega_{i}\left(12 \cdot 10^{3}\right)}=\sqrt{120} \sigma_{\omega_{i}(100)}
$$

Figures 5 and 6 illustrate the mean and the standard deviation of the number of objects retrieved within the deadline $D_{i}=2 \cdot 10^{3}$, compared to the mean and standard deviation predicted by CLT using 12 and 13 .

The probability of retrieving more than $S_{i}$ objects within the deadline $D_{i}$ can be derived using the CDF of the normal distribution using in Equation (9):

$$
\begin{aligned}
\operatorname{Pr}\left(\omega_{i}\left(2 \cdot 10^{3}\right) \geqslant S_{i}\right) & =1-\left[\frac{1}{2}+\frac{1}{2} \operatorname{erf}\left(\frac{\left(S_{i}-1\right)-\mu_{\omega_{i}\left(2 \cdot 10^{3}\right)}}{\sqrt{2} \sigma_{\omega_{i}\left(2 \cdot 10^{3}\right)}}\right)\right] \\
& =1-\left[\frac{1}{2}+\frac{1}{2} \operatorname{erf}\left(\frac{\left(S_{i}-1\right)-120 \mu_{\omega_{i}(100)}}{\sqrt{2} \sqrt{120} \sigma_{\omega_{i}(100)}}\right)\right]
\end{aligned}
$$

By performing 125 ARGoS simulations, Figures 7 and 8 show the probability density function (PDF) associated with the number of objects retrieved by the swarm at deadline $D_{i}=2 \cdot 10^{3}$. In Figure 7 using the mean $\mu_{\omega_{i}(100)}=$ 10.3411 and standard deviation $\sigma_{\omega_{i}(100)}=2.7931$ measured after the system stabilizes and in Figure 8 using the the mean $\mu_{\omega_{i}(100)}=10.6239$ and standard deviation is $\sigma_{\omega_{i}(100)}=2.9755$ measured with taking the system performance though the start-up time into account. Figures 9 and 10 show the cumulative distribution function (CDF) associated with the number of retrieved objects also in Figure 9 after the system stabilizes and in Figure 10 with taking the start-up time into account.

\section{- Single robot performance over short experiments:}

Here, we estimate the swarm performance at deadline $D_{i}=2 \cdot 10^{3}$ in a selforganized way by using the single robot performance measured within one time-window by substituting it in Equation (3).

$$
\omega_{i}\left(D_{i}\right)=\sum_{j=1}^{K} \sum_{l=1}^{N} \beta_{i l}\left(\tau_{j}\right) \quad \Rightarrow \quad \omega_{i}\left(12 \cdot 10^{3}\right)=\sum_{j=1}^{120} \sum_{l=1}^{30} \beta_{i l}(100)
$$

According to the CLT, the r.v. associated with objects retrieved by the swarm up to the deadline $12 \cdot 10^{3}$, is normally distributed with the mean and the standard deviation as in following:

$$
\mu_{\omega_{i}\left(12 \cdot 10^{3}\right)}=120 \times 30 \mu_{\beta_{i j}(100)} \quad \sigma_{\omega_{i}\left(12 \cdot 10^{3}\right)}=\sqrt{120 \times 30} \sigma_{\beta_{i j}(100)}
$$

This estimation can be performed by the robots themselves and could help them in making appropriate allocation decisions. The probability of interest in Equation (4) can be calculated by applying the CDF of normal distribution (9): 


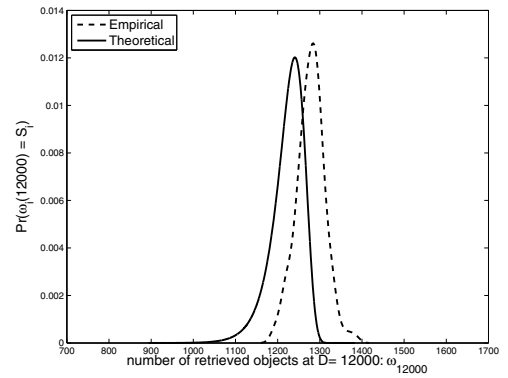

Fig. 7. PDF of the retrieved number of objects without taking the start-up time into account

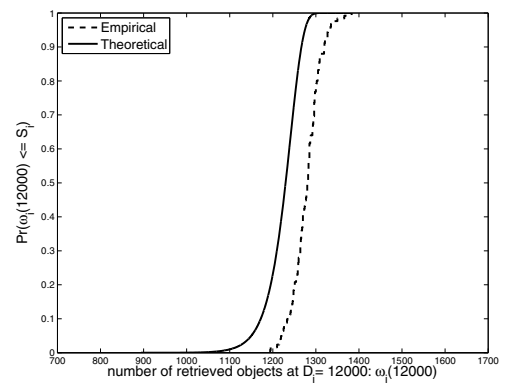

Fig. 9. CDF of the retrieved number of objects without taking the start-up time into account

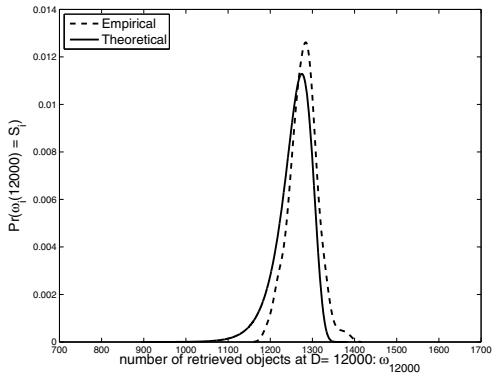

Fig. 8. PDF of the retrieved number of objects with taking the start-up time into account

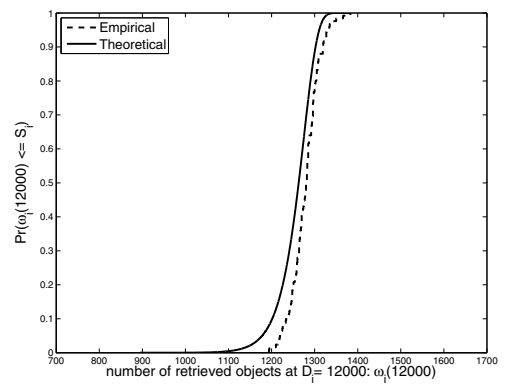

Fig. 10. CDF of the retrieved number of objects with taking the start-up time into account

$$
\begin{aligned}
\operatorname{Pr}\left(\omega_{i}\left(2 \cdot 10^{3}\right) \geqslant S_{i}\right) & =1-\left(\frac{1}{2}+\frac{1}{2} \operatorname{erf}\left(\frac{\left(S_{i}-1\right)-\mu_{\omega_{i}\left(2 \cdot 10^{3}\right)}}{\sqrt{2} \sigma_{\omega_{i}\left(2 \cdot 10^{3}\right)}}\right)\right) \\
& =1-\left(\frac{1}{2}+\frac{1}{2} \operatorname{erf}\left(\frac{\left(S_{i}-1\right)-120 \times 30 \mu_{\beta_{i j}(100)}}{\sqrt{2} \sqrt{120 \times 30} \sigma_{\beta_{i j}(100)}}\right)\right)
\end{aligned}
$$

The swarm performance here is simulated only in the situation of taking the start-up time into account. Measuring the swarm performance after the system stabilizes is straightforward as mentioned above. Figure 11 shows the comparison between the estimated (PDF) of the r.v. associated with the number of objects retrieved by the 30 robot at $D_{i}=2 \cdot 10^{3}$ and the empirical one. Figure 12 shows the same comparison but for the cumulative distribution function(CDF) of both. The reason why the estimation performed using the individual robot's contribution is not the same accurate as the one performed using the swarm contribution, is that, in the case of the swarm the average performance of $N$ robot is taken into account. 


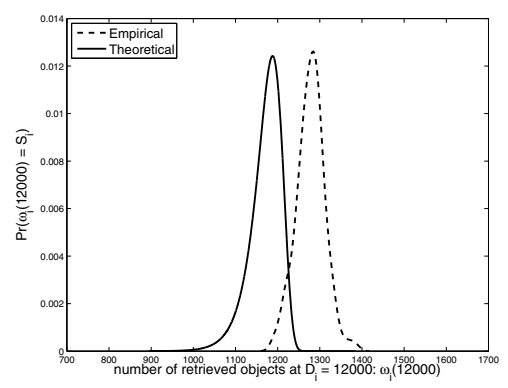

Fig. 11. PDF of the number of retrieved objects estimated based on single robot performance

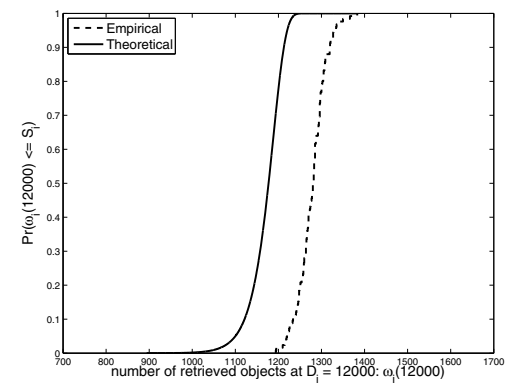

Fig. 12. CDF of the number of retrieved objects estimated based on single robot performance

\section{Conclusion}

In this paper, we presented a probabilistic study of the swarm performance that can be achieved within long-term deadlines and under the influence of spatial interferences. Estimating the performance of robots swarms is an important concept especially for tasks where the performance should be planned under specific constraints like time constraints. In such cases the early estimation of the performance will be obtained at the long-term deadline of the considered task, is of a significant importance.

The central limit theorem CLT, is a straightforward tool and the core theorem which was investigated in this paper to perform the global performance estimation of the swarm. Such a mathematical estimation represents a useful tool to preserve time and resources in comparison to real-time experiments or computer simulations. The estimation of the swarm robotic performance over long-term deadlines using the central limit theorem was accomplished into two contexts: first by using the swarm contributions over short-term experiments and second in a selforganized way by using the single robot performance over short experiments.

The accomplishment of the performance estimation over short experiments, allows for the possibility of launching repair mechanisms at an early stage of the execution. In addition, this estimation is considered to be useful especially in cases where the swarm performance and/or the single robot performance are not following a well-known distribution like the normal distribution. In such cases, the central limit theorem can be applied efficiently to accomplish swarm performance estimations.

\section{References}

1. Goldberg, D.: Evaluating the dynamics of agent-environment interaction. Ph.D. thesis, University of Southern California (2001)

2. Goldberg, D., Matarić, M.J.: Reward maximization in a non-stationary mobile robot environment. In: Proceedings of the fourth International Conference on $\mathrm{Au}$ tonomous Agents, AGENTS 2000, pp. 92-99. ACM, New York (2000) 
3. Jacod, J., Podolskij, M., Vetter, M.: Limit theorems for moving averages of discretized processes plus noise. The Annals of Statistics 38(3), 1478-1545 (2010)

4. Lein, A., Vaughan, R.T.: Adaptive multirobot bucket brigade foraging. In: Proceedings of the Eleventh International Conference on Artificial Life, ALife XI, pp. 337-342. MIT Press (2008)

5. Lerman, K., Galstyan, A.: Mathematical model of foraging in a group of robots: Effect of interference. Autonomous Robots 13(2), 127-141 (2002)

6. Lerman, K., Martinoli, A., Galstyan, A.: A Review of Probabilistic Macroscopic Models for Swarm Robotic Systems. In: Şahin, E., Spears, W.M. (eds.) Swarm Robotics 2004. LNCS, vol. 3342, pp. 143-152. Springer, Heidelberg (2005)

7. Liu, W., Winfield, A.F.T., Sa, J.: Modelling swarm robotic systems: A case study in collective foraging. In: Towards Autonomous Robotic Systems (TAROS 2007), pp. 25-32. Aberystwyth (2007)

8. Martinoli, A., Easton, K., Agassounon, W.: Modeling swarm robotic systems: A case study in collaborative distributed manipulation. Int. Journal of Robotics Research 23, 415-436 (2004)

9. Muniganti, P., Pujol, A.O.: A survey on mathematical models of swarm robotics. In: Workshop of Physical Agents (2010)

10. Ostergaard, E.H., Sukhatme, G.S., Matari, M.J.: Emergent bucket brigading: a simple mechanisms for improving performance in multi-robot constrained-space foraging tasks. In: Proceedings of the Fifth International Conference on Autonomous Agents, AGENTS 2001, pp. 29-30. ACM, New York (2001)

11. Pinciroli, C., Trianni, V., OGrady, R., Pini, G., Brutschy, A., Brambilla, M., Mathews, N., Ferrante, E., Caro, G., Ducatelle, F., Birattari, M., Gambardella, L., Dorigo, M.: Argos: a modular, parallel, multi-engine simulator for multi-robot systems. Swarm Intelligence 6, 271-295 (2012)

12. Pini, G., Brutschy, A., Birattari, M., Dorigo, M.: Interference reduction through task partitioning in a robotic swarm or: dont you step on my blue suede shoes! (2009)

13. Pini, G., Brutschy, A., Birattari, M., Dorigo, M.: Task partitioning in swarms of robots: Reducing performance losses due to interference at shared resources. In: Cetto, J., Filipe, J., Ferrier, J.L. (eds.) Informatics in Control Automation and Robotics. LNEE, vol. 85, pp. 217-228. Springer, Heidelberg (2011)

14. Rice, J.A.: Mathematical Statistics and Data Analysis, 3rd edn. Duxbury Press (April 2001)

15. Shell, D.A., Mataric, M.J.: On foraging strategies for large-scale multi-robot systems. In: IEEE/RSJ International Conference on Intelligent Robots and Systems, pp. 2717-2723. IEEE (2006)

16. Vaughan, A.L.R.T.: Adaptive multi-robot bucket brigade foraging. Artificial Life 11, 337 (2008) 